\title{
Characteristics of double planar thin film inductor with Ni-based magnetic film for DC/DC integration
}

\author{
Mingming Chen ${ }^{1, a}$, Guifu Ding ${ }^{2, b}$ and Mengyuan Zhao ${ }^{3, c}$ \\ ${ }^{123}$ School of Electronic Information and Electrical Engineering \\ Shanghai Jiao Tong University, Shanghai, China \\ accmm0319@sjtu.edu.cn, bgfding@sjtu.edu.cn, czhaomy@sjtu.edu.cn
}

\begin{abstract}
Keywords: double planar, film inductor, micromachining, magnetic thin film Abstract.This paper mainly describes the design, fabrication and test of double planar thin film inductor. The thin film inductor has a sandwich structure, which consists of a double-square spiral planar $\mathrm{Cu}$ coil between top and bottom Ni magnetic thin films on a glass substrate; it is fabricated by dry and wet processes. The inductor is $2.7 \times 1.9 \mathrm{~mm}^{2}$ in area, which is suitable for a $\mathrm{MHz}$ switching DC - DC converter integration. The finally measurement shows that inductance of $0.71 \mathrm{uH}$ constant up to $1.5 \mathrm{MHz}$, and a quality factor of 1.09 at $1.5 \mathrm{MHz}$.
\end{abstract}

\section{Introduction}

As the size and weight of portable electronic products constantly decrease, and its power management chip requirements for internal integration is becoming more and more important. Thin-film magnetic inductors are useful for integrated power delivery solutions for microprocessors and mobile devices[1][2]. In recent years, with the rapid development of MEMS fabrication technology, specially the Quasi LIGA processing technology based on the 3D non-silicon materials becomes one of the most advanced technologies to study on micro-inductive device. Combined the thin-film technology with MEMS technology, miniaturized inductor device is developed, which will have the advantages of having a low resistance, small size, low loss, low cost and mass production. Especially, for power electronics applications, such as switching converters and inverters, planar inductive devices operating in higher frequencies, have many advantages over their conventional counterparts; better thermal management and higher power densities[3][4].

In addition, for the devices with magnetic thin film, the magnetic thin film can be well concentrated magnetic field lines and effectively reducing the eddy current loss of substrate, so loss of the substrate can be ignored here[5]. At the same time, in order to reduce the area occupied by the planar film inductor and compatible with the process, this paper proposed a planar inductor with magnetic shielding film layer structure. What's more, in order to design more number of turns in smaller area, in the design adopted double planar spiral inductors for the structural are designed and optimized.

\section{Design of planar inductor}

Professor S.S Mohaa, through research and analysis, combining with the predecessors' achievements, proposed a simple formula for the square, hexagonal and octagonal inductance calculation [6].

The inductance is found from where $\mathrm{L}$ is the inductance value of the inductor, the unit for $\mathrm{nH}, n$ represents the coil number of turns, $\mu_{0}$ is the magnetic permeability, $d_{\text {avg }}$ is the average of the inductance coil inside and outside diameters, both $\mathrm{K}_{1}$ and $\mathrm{K}_{2}$ are constant, which are determined by the shape of the inductance coil, and $\rho$ represents the fill rates. From (1), it can be seen that in order to achieve large inductance, $n$ should be much larger. Besides the inductance is also related to the size of the coil. At the same time, in order to reduce the area accounted for the planar inductor and compatible with the process, this paper designed a double planar spiral inductor with magnetic shielding layer, which is a sandwich structure. The novel spiral coil structure is shown in Fig. 1. On 
the other hand, this paper also designed the inductor structures with different size to study the effect of inductor with different size on its properties. Sizes and shapes are shown in Table 1.

Table.1 The sizes and shapes of the inductors

\begin{tabular}{cccccccccc}
\hline shape & \multicolumn{3}{c}{ square } & \multicolumn{3}{c}{ circle } \\
\hline $\begin{array}{c}\text { Coil inner area } \\
\left(\mathrm{mm}^{\wedge} 2\right)\end{array}$ & $0.11^{*} 0.08$ & & $0.15^{*} 0.1$ & & $\mathrm{Pi}^{*} 0.1^{\wedge} 2$ \\
\hline spacing (um) & & & & & & & & \\
\hline Line width (um) & 20 & 30 & 40 & 20 & 30 & 40 & 20 & 30 & 40 \\
\hline $\begin{array}{c}\text { Number of turns } \\
\text { (single layer) }\end{array}$ & 17 & 14 & 12 & 18 & 14 & 12 & 17 & 13 & 11 \\
\hline type & A & B & C & D & E & F & G & H & I \\
\hline
\end{tabular}

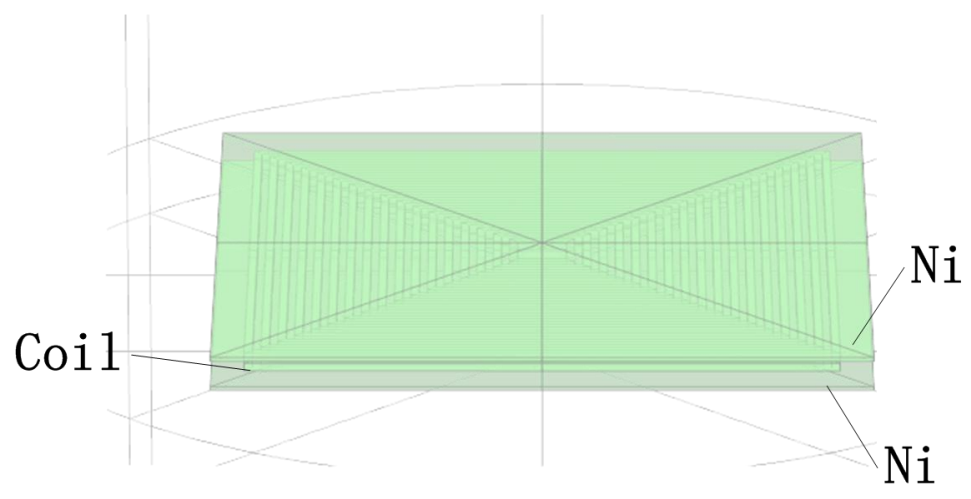

Fig.1 The structure of magnetic thin film inductor

\section{Fabrication}

There exist some of the key steps for fabricating the thin film inductor, such as sputtering, photolithography and electroplating. During the MEMS fabrication, it addressed the following key manufacturing technology. 1) Through rational design and double-sided mask overlay alignment mark and multilayer depth lithography technology to solve the question in the multi-layer mask overlay process and get plating resist mold, to obtain good aspect ratio resist mold. 2) By exerting additive in the plating solution, to achieve a high aspect ratio micro-sized plating issues. 3) Polyimide resolved the insulation issue between the Micro inductor layers and the micro interturn. After coating polyimide and the drying and curing process, then precision mechanical polishing, the substrate can be guaranteed to have a flat surface. Through the above processes, the structure of the proposed thin film inductor with two same spirals, which have 17 turns. In order to improve the inductance of the thin film inductor, the connection between two coils is in series by a through-hole. The $10 \mathrm{um}$ thickness Ni magnetic thin films for the top and bottom magnetic layers were electroplated by Ni. A $20 \mathrm{um}$ thickness ofCu film for the thin film coil was also prepared by electroplating technology. The single coil structure is shown in fig.2. Fig. 3 is a SEM image of an example of the fabricated magnetic thin film inductors. Dimensions of the thin film inductors fabricated in this work are listed in Table I. 

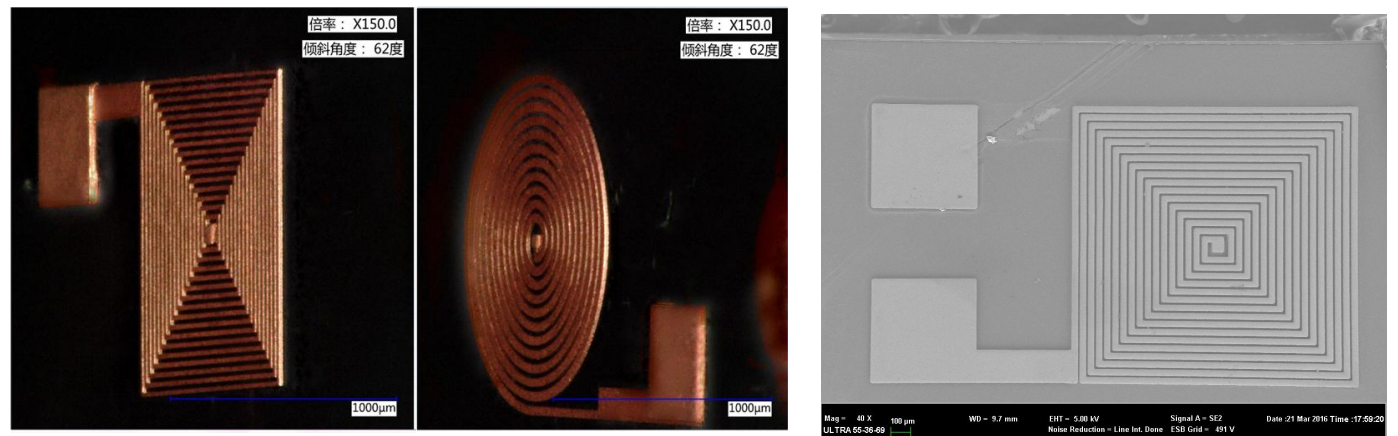

Fig.2 3D structure of the single coil

Fig.3 Example of SEM image of fabricated magnetic thin

\section{Results and conclusion}

The frequency characteristics of the thin film inductors were measured by Agilent E4991A Rf impedance material analyzer, at $0.5 \mathrm{~V}$. Fig. 4(a) -(c) indicate the inductance L of several kinds of magnetic thin film inductors (type A to I in the figure). With an increase of the line width, the total inductance of the fabricated inductor decreases due to the self and mutual inductance decrease.

The inductance $\mathrm{L}$ was nearly proportional to the number of turns of the inductor. The smallest width of device line with square (type D; 20um of the line width and $0.15^{*} 0.1 \mathrm{~mm}^{\wedge} 2$ of Coil inner area) had a $1 \mathrm{uH}$ constant inductance up to $128.8 \mathrm{kHz}$. However, Fig. 5(a) -(c) indicates the frequency characteristics of quality factor of several kinds of magnetic thin film inductors (type A to I in the figure). The quality factor was not definite to the number of turns of the inductor. The quality factor reflects the performance of the inductor. However, the high inductance and high quality factor can't be achieved at the same time. the inductor must compromise between them.

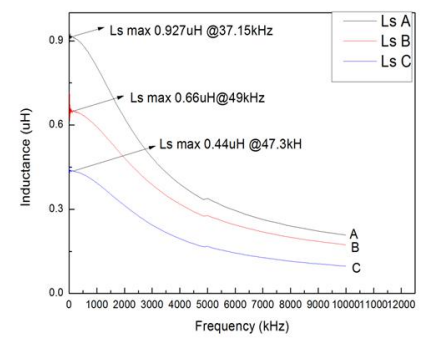

(a)

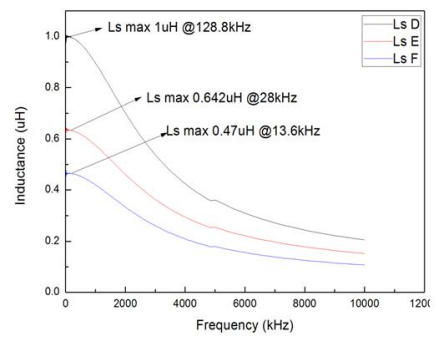

(b)

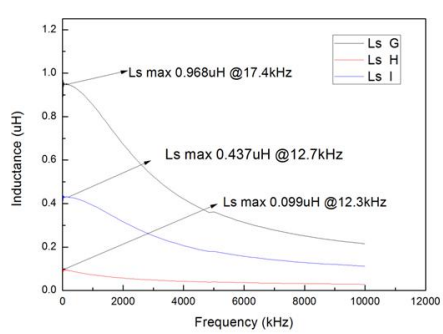

(c)

Fig. 4. the inductance $\mathrm{L}$ of several kinds of magnetic thin film inductors

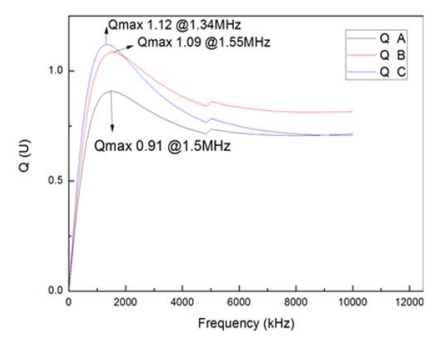

(a)

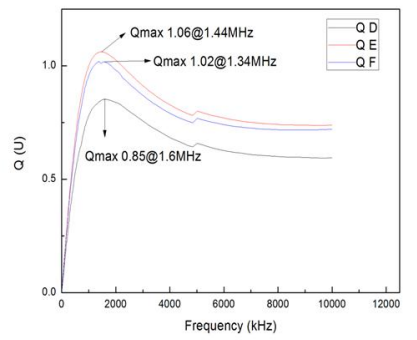

(b)

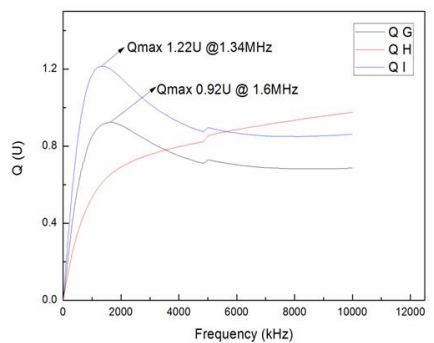

(c)

Fig. 5. the quality factor of several kinds of magnetic thin film inductors

The twin spiral type thin film inductor with $\mathrm{Ni}$ magnetic thin films is fabricated and its characteristics are investigated. This paper represents a suitable for the miniaturization of DC - DC converter integrated micro thin film inductor in a $2.7 \times 1.9 \mathrm{~mm}^{2}$ size. The inductance was about $0.77 \mathrm{uH}$ at $1.5 \mathrm{MHz}$ and the quality factor was about 1 (type $\mathrm{B}$; the line width with $30 \mathrm{um}$ and coil inner area with $0.11 * 0.1 \mathrm{~mm}^{\wedge} 2$ ). The magnetic flux inductance offsets with each other is the main limiting factor to the quality of the on-chip spiral inductors. 


\section{References}

[1] Elsherbini A, Braunisch H, O'Brien K. Circuit modeling of nonlinear lossy frequency dependent thin-film magnetic inductors[C]// Electrical Performance of Electronic Packaging and Systems (EPEPS), 2012 IEEE 21st Conference on. IEEE, 2012:105-108.

[2] Sato F, Ono T, Wako N, et al. All-in-one package ultracompact micropower module using thin-film inductor[J]. Magnetics IEEE Transactions on, 2004, 40(4):2029-2031.

[3] Sato T, Tomita H, Sawabe A, et al. A magnetic thin film inductor and its application to a $\mathrm{MHz}$ switching dc-dc converter[J]. Magnetics IEEE Transactions on, 1994, 30(2):217-223.

[4] Rais-Zadeh M, Kohl P A, Ayazi F. MEMS Switched Tunable Inductors[J]. Journal of Microelectromechanical Systems, 2008, 17(1):78-84.

[5] Yamaguchi M, Suezawa K, Arai K I, et al. Microfabrication and characteristics of magnetic thin-film inductors in the ultrahigh frequency region[J]. Journal of Applied Physics, 1999, 85(11):7919-7922.

[6] Maksimovic D. Fundamentals of Power Electronics / R.W. Erickson, D. Maksimovic.[J]. Springer Netherlands, 2001, 35(1):1.1-1.9. 